\title{
Immunodeficiency in Children Starting Antiretroviral Therapy in Low-, Middle-, and High-Income Countries
}

\author{
Manuel Koller, DrSc, * Kunjal Patel, DSc, † Benjamin H. Chi, MD, $\neq$ Kara Wools-Kaloustian, MD, $\S$ \\ Fatoumata Dicko, MD,\| Kulkanya Chokephaibulkit, MD, $₫$ Cleophas Chimbetete, MD,\# \\ Dorita Avila, MSc, * Rohan Hazra, MD, ** Samual Ayaya, MD, †† Valeriane Leroy, MD, PhD, $+\neq$ \\ Huu Khanh Truong, MD, §§ Matthias Egger, MD, * ||| and Mary-Ann Davies, MD, PhD, ||| for the IeDEA, \\ NISDI, PHACS and IMPAACT 219C studies
}

\begin{abstract}
Background: The CD4 cell count or percent (CD4\%) at the start of combination antiretroviral therapy (cART) is an important prognostic factor in children starting therapy and an important indicator of program performance. We describe trends and determinants of CD4 measures at cART initiation in children from low-, middle-, and high-income countries.
\end{abstract}

\begin{abstract}
Methods: We included children aged $<16$ years from clinics participating in a collaborative study spanning sub-Saharan Africa, Asia, Latin America, and the United States. Missing CD4 values at cART start were estimated through multiple imputation. Severe immunodeficiency was defined according to World Health Organization criteria. Analyses used generalized additive mixed models adjusted for age, country, and calendar year.
\end{abstract}

Results: A total of 34,706 children from 9 low-income, 6 lower middle-income, 4 upper middle-income countries, and 1 high-income country (United States) were included; 20,624 children (59\%) had severe immunodeficiency. In low-income countries, the estimated prevalence of children starting cART with severe immunodeficiency declined from $76 \%$ in 2004 to $63 \%$ in 2010 . Corresponding figures for lower middle-income countries were from $77 \%$ to $66 \%$ and for upper middle-income countries from $75 \%$ to $58 \%$. In the United States, the percentage decreased from $42 \%$ to $19 \%$ during the period 1996 to 2006. In low- and middle-income countries, infants and children aged 12-15 years had the highest prevalence of severe immunodeficiency at cART initiation.

Conclusions: Despite progress in most low- and middle-income countries, many children continue to start cART with severe

Received for publication March 4, 2014; accepted August 29, 2014.

From the *Institute of Social \& Preventive Medicine (ISPM), University of Bern, Bern, Switzerland; $†$ Department of Epidemiology, Harvard School of Public Health, Boston, MA; †Centre for Infectious Disease Research in Zambia, Lusaka, Zambia; §Department of Medicine, Indiana University School of Medicine, Indianapolis, IN; ||Department of Pediatrics, Gabriel Toure Hospital, Bamako, Mali; qFaculty of Medicine Siriraj Hospital, Mahidol University, Bangkok, Thailand; \#Newlands Clinic, Harare, Zimbabwe; **Maternal and Pediatric Infectious Disease Branch, Eunice Kennedy Shriver National Institute of Child Health and Human Development (NICHD), Bethesda, MD; ††Department of Pediatrics, College of Health Sciences, Moi University, Kenya; $+ \pm I N S E R M$, French National Institute for Health and Medical Research, U897, Bordeaux, France; §§Children's Hospital 1, Ho Chi Minh City, Vietnam; and ||||School of Public Health and Family Medicine, University of Cape Town, Cape Town, South Africa.

The African regions of the International epidemiologic Databases to Evaluate AIDS (IeDEA) are supported by the National Cancer Institute (NCI), the Eunice Kennedy Shriver National Institute of Child Health and Human Development (NICHD), and the National Institute of Allergy And Infectious Diseases (NIAID) as part of the International epidemiologic Databases to Evaluate AIDS (IeDEA) (Grants 5U01AI069919-04, 5U01-AI069924-05, 1U01 AI069927, and U01AI069911-01). The Treat Asia pediatric HIV Observational Database is an initiative of TREAT Asia, a program of amfAR, The Foundation for AIDS Research, with support from AIDS Life Austria and NIAID, NICHD, and NCI, as part of IeDEA (U01AI069907). The Kirby Institute is funded by the Australian Government Department of Health and Aging and is affiliated with the Faculty of Medicine, University of New South Wales. The NICHD Site Development Initiative (NISDI) was funded by the NIH and NICHD (contracts N01-HD-3-3345 and N01-HD-8-0001). The Pediatric HIV/AIDS Cohort Study (PHACS) was supported by the Eunice Kennedy Shriver National Institute of Child Health and Human Development with co-funding from the National Institute on Drug Abuse, the National Institute of Allergy and Infectious Diseases, the Office of AIDS Research, the National Institute of Mental Health, the National Institute of Neurological Disorders and Stroke, the National Institute on Deafness and Other Communication Disorders, the National Heart Lung and Blood Institute, the National Institute of Dental and Craniofacial Research, and the National Institute on Alcohol Abuse and Alcoholism, through cooperative agreements with the Harvard University School of Public Health (HD052102, 3 U01 HD052102-05S1, and 3 U01 HD052102-06S3) and the Tulane University School of Medicine (HD052104, 3U01HD052104-06S1). Support for the International Maternal Pediatric Adolescent AIDS Clinical Trials Group (IMPAACT) 219C study is provided by the NIAID (U01 AI068632) and NICHD (contract N01-3-3345 and HHSN267200800001C). This work was also supported by the Statistical and Data Analysis Center at Harvard School of Public Health, under the NIAID cooperative agreement \#5 U01 AI41110 with the Pediatric AIDS Clinical Trials Group (PACTG) and \#1 U01 AI068616 with the IMPAACT Group.

Presented at the 20th Conference on Retroviruses and Opportunistic Infections, March 3-7, 2013, Atlanta, GA.

The authors have no conflicts of interest to disclose.

Supplemental digital content is available for this article. Direct URL citations appear in the printed text and are provided in the HTML and PDF versions of this article on the journal's Web site (www.jaids.com).

The content of this publication is solely the responsibility of the authors and does not necessarily represent the official views of any of the institutions and funders mentioned.

Correspondence to: Mary-Ann Davies, MD, PhD, School of Public Health and Family Medicine, University of Cape Town Faculty of Health Sciences, Anzio Road, Observatory, Cape Town, 7925, South Africa (e-mail: mary-ann.davies@uct.ac.za).

Copyright $(\subset 2014$ by Lippincott Williams \& Wilkins 
immunodeficiency. Early diagnosis and treatment of HIV-infected children to prevent morbidity and mortality associated with immunodeficiency must remain a global public health priority.

Key Words: children, antiretroviral, immunodeficiency, CD4, global (J Acquir Immune Defic Syndr 2015;68:62-72)

\section{INTRODUCTION}

Access to combination antiretroviral therapy (cART) has increased dramatically since 2004, with substantial funding from donors and national governments. According to World Health Organization (WHO) estimates, the number of children receiving CART in low- and middle-income countries increased from 71,500 at the end of 2005 to 740,000 in 2013, an increase in coverage among all HIV-infected children from $11 \%$ to $23 \%$. ${ }^{1,2}$ However, in many settings, mortality remains high during the first year of cART, especially among children with advanced disease. ${ }^{3-6}$

Immunologic status (CD4 cell count or percentage), age, and clinical stage at cART initiation are important prognostic factors for $\mathrm{HIV}$-infected children and WHO guidelines for starting therapy have been based on these criteria. ${ }^{7}$ A recent prognostic model from Southern Africa estimated that mortality in the first year of treatment in children starting cART with CD4\% $\geq 10 \%$ was about half that of children with $\mathrm{CD} 4 \%<5 \%{ }^{8}$ Similarly, mortality in children with WHO clinical stage $3 / 4$ was about $40 \%$ higher compared with children in stages $1 / 2$, and about 4 times higher in infants compared with children $5-10$ years. $^{8}$

Until recently, WHO guidelines recommended immediate cART regardless of immunologic or clinical thresholds for infants and children aged $<2$ years and for all children aged 2 5 years with WHO clinical stages $3 / 4$, or CD4 $<750$ cells per microliter or $25 \%{ }^{7}$ These guidelines were revised in 2013 to recommend immediate treatment for all children aged $<5$ years. ${ }^{9}$ Previous studies indicate that most children in lower income settings do not initiate cART until they have severe immunosuppression. Among 10,875 children who initiated treatment from 2004 to 2010 in Malawi, South Africa, Zambia, and Zimbabwe, most had advanced disease $(72 \%$ WHO stage 3/4) with low median CD4\% (13\%). ${ }^{8}$ Temporal trends in CD4 measures at cART initiation are a useful indicator of the ability of health care systems to identify and treat eligible children in a timely fashion and to respond to changing guidelines. We analyzed these indicators from an international collaboration of treatment programs in sub-Saharan Africa, Asia, Latin America, and North America.

\section{METHODS}

\section{Data Sources}

The International epidemiologic Databases to Evaluate AIDS (IeDEA) is a global consortium structured through regional centers to pool data on HIV-infected individuals. The 7 regions included in IeDEA are North America, Caribbean/ Central and South America, Asia/Pacific, and 4 regions in subSaharan Africa (East Africa, West Africa, Central Africa, and
Southern Africa). ${ }^{6,10-14}$ For the present analysis, we included data from the African and Asia/Pacific regions of IeDEA. In addition, we included North American and Latin American data from the NICHD International Site Development Initiative, ${ }^{15}$ the Pediatric HIV/AIDS Cohort Study, ${ }^{16}$ and the International Maternal Pediatric Adolescent AIDS Clinical Trials 219C study. ${ }^{17}$ Pooling of data and their use in collaborative analyses were approved by local ethics committees and institutional review boards. Participating regional centers sent deidentified data to the University of Bern, Switzerland, where the data were collated and analyzed.

\section{Inclusion Criteria and Definitions}

All patients aged $<16$ years with documented gender and cART start date were included in descriptive analyses of age, gender, and calendar year at cART initiation. For further analyses we excluded data from low or middle-income countries before widespread rollout began in 2003, data from calendar years from any country with $<10$ children with CD4 measures for that year and countries contributing $<50$ children with CD4 measures overall after applying the calendar year exclusion criterion. cART was defined as a regimen of $\geq 3$ antiretroviral drugs, typically from 2 drug classes. Baseline CD4 values were defined as those nearest to cART start date, within a window of 6 months before to 1 month after treatment initiation. Countries were grouped according to the World Bank classification of Gross National Income per capita per year in 2010 as low income (LIC) ( $\leq$ US\$1005), lower middle income (LMIC) (US\$1006 to 3975), upper middle income (UMIC) (US\$3976 to 12,275), and high income (HIC) ( $\geq$ US\$12,276). ${ }^{18}$ Age groups were $<12$ months, 12-35 months, 36-59 months, 5-11 years, and 12-15 years. Severe immunodeficiency was defined according to WHO criteria as CD4\% $<25 \%$ (age $<12$ months), $<20 \%$ (age 12-35 months), $<15 \%$ (age $36-59$ months), and CD4 count $<200$ cells per microliter or CD $4 \%<15 \%$ (age $\geq 5$ years). ${ }^{19}$

\section{Descriptive Analyses}

Descriptive analyses included all countries and calendar years with any data from 1995 onwards. Analyses were stratified by country, age group, gender, and country income group. CD4 values, age, and calendar year at cART start were summarized as medians with interquartile ranges (IQRs) or percentages.

\section{Multiple Imputation of Missing CD4 Measures}

We imputed missing CD4 measures for countries contributing $\geq 50$ children with CD4 measures and calendar years with $\geq 10$ children with CD4 measures. We imputed log of CD4\% and count simultaneously using chained equations and predictive mean matching, adjusting for country, year of cART start, continent and national cART coverage, and stratifying by gender, age, and income group. Data on pediatric cART coverage for 2009 were obtained from WHO for low- and 
middle-income countries. ${ }^{20}$ We generated 50 imputed data sets and combined these using Rubin's rule. ${ }^{21}$

\section{Analyses of Temporal Trends in CD4 Measures}

We used generalized additive mixed models to analyze temporal trends in CD4 measures in different age and country income groups. We analyzed the proportion of children with severe immunodeficiency, median CD4\% (aged $<5$ years), and median CD4 count (aged $\geq 5$ years). Gender, age group and income group, as well as their interactions were entered as fixed effects. Yearly trends were smoothed by gender, age group, and income group. The data were aggregated by calendar year (3-12 years, depending on country), country (20 countries), gender (2 groups), and age group (5 groups, as defined above) before analysis: each combination of these factors corresponded to a cell in the analysis. For each cell, we calculated the number of children with severe immunodeficiency and the median CD4\% and count. Each cell was entered in the model with a weight corresponding to the number of observations it contained divided by the average number of observations in all cells. We modeled the prevalence of severe immunodeficiency using the binomial distribution and a logit link, assuming Gaussian distributions for the error terms of CD4\% and count. We used the same modeling technique to model temporal trends in age at cART start in different country income groups, however, did not stratify by age group and restricted to years where all age groups were represented.

In an analysis restricted to low- and middle-income countries with data for the year 2009, we modeled the country-level proportion of patients starting cART with severe immunodeficiency using a generalized linear mixed model. We chose the year 2009 because this was the most recent year where data from most countries were available. We examined the influence of age, gender, income group, and national pediatric cART coverage. These results are presented as adjusted odds ratios (ORs) with 95\% confidence intervals (CIs).

We used the data set with imputed data for the main analysis. In sensitivity analyses, we fitted the model to complete data. Analyses were done in Stata 12 (Stata Corporation, College Station, TX) and R version 3.0.2 (R Core Team, Vienna, Austria). The technical appendix provides further details on the multiple imputation and smoothing (see Supplemental Digital Content 1, http://links.lww.com/QAI/A579). CD4 results are presented as observed or modeled median (IQR) CD4 count $/ \%$ and proportion of children with severe immunodeficiency with $95 \% \mathrm{CI}$.

\section{RESULTS}

\section{Descriptive Analyses}

Data of 36,125 children aged $<16$ years were submitted to the data center. Nine countries with $\leq 200$ children, 7 with 201-1000 children, 7 with 1001-5000 children, and 2 with $>5000$ children contributed data (Fig. 1). Ten children with missing gender were excluded; hence 36,115 children from 25 countries were included in descriptive analyses. Supplementary Figure S1 (see Supplemental Digital Content 2, http://links.lww.com/QAI/A579) shows the inclusion of children in analyses.

The number of children included from each country varied from 30 (Peru) to 11,830 (Zambia) (Table 1). The percentage of girls was $49 \%$ overall [range across countries $40 \%$ (Senegal) to $54 \%$ (Botswana)]. Median age at cART initiation was 5.2 years [range, 10 months (Peru) to 9.1 years (Zimbabwe)]. The median year of cART initiation was 2007 [range, 1998 (United States) to 2008 (Peru, Vietnam, Zambia, and Zimbabwe)]. The percentage of children starting cART with severe immunodeficiency was $57 \%$ overall $(20,619$ children), range, 22\% (United States) to $83 \%$ (Peru) (Table 2).

The median (IQR) age of children starting cART was 5.6 years (2.4-9.4) in LIC, 5.3 (2.1-9.6) in LMIC, 4.5 (1.58.0) in UMIC, and 6.0 (3.0-10.0) in HIC. The median CD4 count at cART initiation in children aged $\geq 5$ years was 238 cells per microliter in girls and 220 cells per microliter in boys. In LIC, it was 226 cells per microliter (girls) and 206 cells per microliter (boys). Corresponding values were higher in LMIC (246 and 236 cells/ $\mu \mathrm{L}$, respectively) but lower in UMIC countries (191 and 183 cells/ $\mu \mathrm{L}$, respectively). In the United States, the values were 468 and 414 cells per microliter, respectively. Similar patterns were evident for CD4\% in children $<5$ years (Table 2 ). The percentage of children starting cART with severe immunodeficiency was $57 \%$ overall. Compared with LIC (58\%), it was slightly lower in LMIC $(56 \%)$, higher in UMIC $(64 \%)$, and lower in the United States $(22 \%)$. Apart from the comparison of age in LIC and HIC, all the above differences were statistically significant $(P<0.05)$. However, such significant differences are to be expected given the large number of observations.

\section{Multiple Imputation of Missing CD4 Measures}

A total of 1409 children were excluded from further analyses, either because they were from Benin, Burkina Faso, India, Botswana, or Peru, with $<50$ children with CD4 measures (413 children) or from calendar years with $<10$ children with CD4 measures (996 children). The multiple imputation of missing CD4 measures was thus based on 34,706 children. Compared with 6528 children who had both measures missing, the 28,178 children with either CD4 count or percent measures available at cART initiation were older, more likely to be in WHO stage $1 / 2$ or CDC stage $\mathrm{A} / \mathrm{B}$, more likely to have information available on clinical stage, and less likely to be from a LIC (see Table S1, Supplemental Digital Content 3, http://links.lww.com/QAI/A579). Both measures were missing in $19 \%$ of children overall, $25 \%$ in LIC, $18 \%$ in LMIC, $17 \%$ in UMIC, and $0 \%$ in the United States (Table 2). Both measures were available in $31 \%$ of children in LIC, $3 \%$ in LMIC, $13 \%$ in UMIC, and $100 \%$ in the United States. Supplementary Figure S2 (see Figure S2, Supplemental Digital Content 4, http://links.lww.com/QAI/A579/) shows the proportion of children with available CD4 measure by country income and age groups. 
FIGURE 1. Map of countries contributing patients to the collaborative analysis.

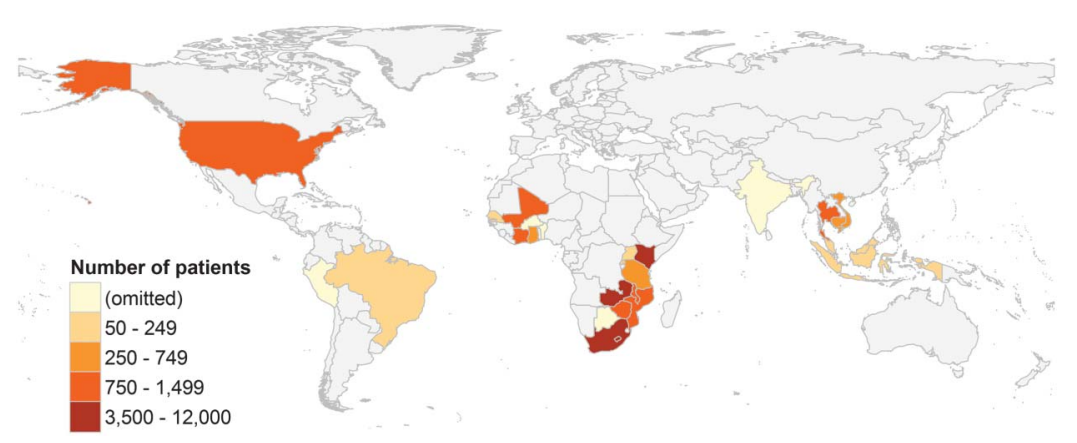

CD4 counts were imputed for 3014 (17\%) of 17,860 children aged $\geq 5$ years with no CD4 measure and for 4017 (22\%) children with only CD4\% available. Similarly, CD4\% values were imputed for 3514 (21\%) of 16,846 children aged $<5$ years with no CD4 measure and for 6514 (39\%) children who had a CD4 count available. Medians of imputed CD4 data (main analysis) and complete cases (sensitivity analysis) were generally similar (Table 2). The CD4 cell counts in UMIC increased somewhat after imputation of missing data, and the percentage of children starting cART with severe immunodeficiency increased in LIC. For children aged $\geq 5$ years, the differences between observed and imputed CD4 values for individual countries ranged from -11 cells per microliter (boys from Mozambique) to +57 cells per

TABLE 1. Characteristics of 36,115 Children Starting cART by Country and World Bank Income Group

\begin{tabular}{|c|c|c|c|c|c|c|c|c|}
\hline \multirow[b]{2}{*}{ Country } & \multicolumn{2}{|c|}{ No. Children } & \multicolumn{2}{|c|}{ Median Age in Yrs } & \multicolumn{2}{|c|}{ Median Calendar Yr of Starting cART } & \multirow{2}{*}{$\begin{array}{c}\text { Calendar Yr Range } \\
\text { of Available Data }\end{array}$} & \multirow{2}{*}{$\begin{array}{c}\text { Calendar Yrs } \\
\text { Included in Modeling* }\end{array}$} \\
\hline & Female & Male & Female & Male & Female & Male & & \\
\hline \multicolumn{9}{|l|}{ Low income } \\
\hline Benin & 33 & 38 & 2.9 & 4.8 & 2004 & 2005 & $2002-2006$ & Excluded \\
\hline Burkina Faso & 72 & 89 & 6.3 & 6.3 & 2005 & 2004 & $2002-2007$ & Excluded \\
\hline Cambodia & 166 & 175 & 5.9 & 6.1 & 2006 & 2006 & $2002-2010$ & 2005-2009 \\
\hline Kenya & 1859 & 2024 & 5.1 & 4.9 & 2007 & 2007 & 2002-2009 & $2003-2008$ \\
\hline Malawi & 729 & 682 & 7.5 & 7.8 & 2008 & 2007 & $2002-2010$ & $2005-2010$ \\
\hline Mali & 321 & 470 & 4.0 & 4.0 & 2006 & 2005 & 2001-2008 & 2003-2008 \\
\hline Mozambique & 567 & 607 & 3.2 & 3.3 & 2006 & 2006 & $1998-2010$ & 2004-2008 \\
\hline Rwanda & 95 & 90 & 7.0 & 7.0 & 2006 & 2006 & 2005-2009 & 2005-2009 \\
\hline Tanzania & 172 & 152 & 8.2 & 6.6 & 2007 & 2007 & $2005-2008$ & $2005-2008$ \\
\hline Uganda & 114 & 109 & 2.7 & 3.3 & 2005 & 2005 & 2003-2009 & 2003-2007 \\
\hline Zimbabwe & 660 & 616 & 9.1 & 9.2 & 2008 & 2008 & 1999-2010 & 2004-2010 \\
\hline Overall (IQR) & 4788 & 5052 & $5.7(2.5-9.7)$ & $5.4(2.4-9.2)$ & 2007 (2005-2008) & 2007 (2005-2008) & 1998-2010 & $2003-2010$ \\
\hline \multicolumn{9}{|c|}{ Lower middle income } \\
\hline Côte d'Ivoire & 618 & 715 & 5.9 & 5.6 & 2005 & 2005 & $2003-2008$ & 2004-2007 \\
\hline Ghana & 164 & 161 & 5.7 & 5.2 & 2007 & 2006 & 2004-2009 & $2005-2008$ \\
\hline India & 42 & 57 & 8.0 & 7.2 & 2006 & 2006 & $2002-2010$ & Excluded \\
\hline Indonesia & 63 & 79 & 2.0 & 1.8 & 2007 & 2007 & $1997-2010$ & $2005-2009$ \\
\hline Senegal & 74 & 111 & 6.4 & 4.5 & 2006 & 2006 & 2000-2009 & $2005-2007$ \\
\hline Vietnam & 198 & 261 & 4.1 & 3.8 & 2008 & 2008 & $2005-2010$ & $2005-2010$ \\
\hline Zambia & 5932 & 5898 & 5.7 & 5.2 & 2008 & 2008 & $2003-2010$ & 2004-2010 \\
\hline Overall (IQR) & 7091 & 7282 & $5.6(2.1-9.7)$ & $5.1(2.1-9.5)$ & 2008 (2006-2009) & 2007 (2006-2009) & $1997-2010$ & 2004-2010 \\
\hline \multicolumn{9}{|c|}{ Upper middle income } \\
\hline Botswana & 28 & 24 & 8.3 & 8.3 & 2003 & 2004 & $1998-2007$ & Excluded \\
\hline Brazil & 84 & 82 & 1.4 & 1.5 & 2006 & 2006 & 2003-2010 & 2004-2009 \\
\hline Malaysia & 100 & 118 & 3.9 & 3.5 & 2006 & 2006 & $2000-2010$ & $2005-2010$ \\
\hline Peru & 14 & 16 & 0.6 & 1.1 & 2008 & 2008 & 2006-2009 & Excluded \\
\hline South Africa & 4276 & 4386 & 4.0 & 3.9 & 2006 & 2006 & $1998-2010$ & $2003-2010$ \\
\hline Thailand & 724 & 646 & 8.3 & 7.5 & 2005 & 2004 & $1999-2010$ & $2003-2010$ \\
\hline Overall (IQR) & 5226 & 5272 & $4.6(1.5-8.3)$ & $4.4(1.5-7.6)$ & $2006(2005-2007)$ & 2006 (2005-2007) & $1998-2010$ & $2003-2010$ \\
\hline \multicolumn{9}{|l|}{ High income } \\
\hline United States & 731 & 673 & 7.0 & 6.0 & 1998 & 1998 & $1995-2009$ & $1995-2006$ \\
\hline Overall (IQR) & 731 & 673 & $7.0(3-10)$ & $6.0(3-9)$ & 1998 (1997-2000) & 1998 (1997-1999) & 1995-2009 & 1995-2006 \\
\hline
\end{tabular}

*Calendar years were excluded from further analyses if there were $<10$ children starting cART with a CD4 cell measure. Five countries were excluded from further analyses because there were $<50$ children with a CD4 cell measure. 
TABLE 2. CD4 Cell Count and CD4\% at the Start of CART and the Percentage of Children Starting CART With Severe Immunodeficiency by Country and World Bank Income Group

\begin{tabular}{|c|c|c|c|c|c|c|}
\hline \multirow[b]{3}{*}{ Country } & \multirow{2}{*}{\multicolumn{2}{|c|}{$\begin{array}{c}\text { Percentage of Children Missing Both CD4 Count } \\
\text { and CD4\% Measurements }\end{array}$}} & \multicolumn{4}{|c|}{$\begin{array}{c}\text { Median CD4 Cell Count at the Start of cART in Cells } / \mu \mathrm{L} \text { of Children } 5 \text { Yrs } \\
\text { or Older }\end{array}$} \\
\hline & & & \multicolumn{2}{|c|}{ Complete Case } & \multicolumn{2}{|c|}{ Imputed Data } \\
\hline & Female & Male & Female & Male & Female & Male \\
\hline \multicolumn{7}{|l|}{ Low income } \\
\hline Benin & $3 \%$ & $0 \%$ & $78 \%$ & $181 \%$ & Excluded & Excluded \\
\hline Burkina Faso & $96 \%$ & $96 \%$ & $108 \%$ & $501 \%$ & Excluded & Excluded \\
\hline Cambodia & $2 \%$ & $5 \%$ & $180 \%$ & $222 \%$ & $170 \%$ & $174 \%$ \\
\hline Kenya & $28 \%$ & $27 \%$ & $261 \%$ & $236 \%$ & $261 \%$ & $236 \%$ \\
\hline Malawi & $48 \%$ & $52 \%$ & $253 \%$ & $209 \%$ & $253 \%$ & $198 \%$ \\
\hline Mali & $4 \%$ & $5 \%$ & $174 \%$ & $157 \%$ & $172 \%$ & $167 \%$ \\
\hline Mozambique & $20 \%$ & $20 \%$ & $299 \%$ & $319 \%$ & $296 \%$ & $325 \%$ \\
\hline Rwanda & $25 \%$ & $30 \%$ & $293 \%$ & $259 \%$ & $265 \%$ & $256 \%$ \\
\hline Tanzania & $42 \%$ & $41 \%$ & $130 \%$ & $117 \%$ & $132 \%$ & $121 \%$ \\
\hline Uganda & $28 \%$ & $28 \%$ & $692 \%$ & $464 \%$ & $635 \%$ & $471 \%$ \\
\hline Zimbabwe & $22 \%$ & $22 \%$ & $186 \%$ & $174 \%$ & $186 \%$ & $174 \%$ \\
\hline Overall (IQR) & $28 \%$ & $28 \%$ & $226 \%(89-404)$ & $206 \%(68-385)$ & $230 \%(94-405)$ & $205 \%(72-388)$ \\
\hline \multicolumn{7}{|c|}{ Lower middle income } \\
\hline Côte d'Ivoire & $16 \%$ & $19 \%$ & $283 \%$ & $288 \%$ & $285 \%$ & $295 \%$ \\
\hline Ghana & $38 \%$ & $42 \%$ & $174 \%$ & $108 \%$ & $155 \%$ & $125 \%$ \\
\hline India & $7 \%$ & $14 \%$ & $192 \%$ & $197 \%$ & Excluded & Excluded \\
\hline Indonesia & $0 \%$ & $6 \%$ & $78 \%$ & $53 \%$ & $78 \%$ & $38 \%$ \\
\hline Senegal & $47 \%$ & $56 \%$ & $78 \%$ & $261 \%$ & $65 \%$ & $297 \%$ \\
\hline Vietnam & $9 \%$ & $11 \%$ & $111 \%$ & $122 \%$ & $104 \%$ & $112 \%$ \\
\hline Zambia & $19 \%$ & $17 \%$ & $250 \%$ & $241 \%$ & $253 \%$ & $241 \%$ \\
\hline Overall (IQR) & $1 \%$ & $18 \%$ & $246 \%(113-422)$ & $236 \%(100-414)$ & $251 \%(116-432)$ & $241 \%(103-422)$ \\
\hline \multicolumn{7}{|c|}{ Upper middle income } \\
\hline Botswana & $18 \%$ & $21 \%$ & $216 \%$ & $326 \%$ & Excluded & Excluded \\
\hline Brazil & $0 \%$ & $0 \%$ & $440 \%$ & $514 \%$ & $452 \%$ & $514 \%$ \\
\hline Malaysia & $20 \%$ & $17 \%$ & $50 \%$ & $103 \%$ & $49 \%$ & $121 \%$ \\
\hline Peru & $0 \%$ & $0 \%$ & NA & NA & Excluded & Excluded \\
\hline South Africa & $17 \%$ & $19 \%$ & $233 \%$ & $238 \%$ & $233 \%$ & $241 \%$ \\
\hline Thailand & $16 \%$ & $17 \%$ & $107 \%$ & $64 \%$ & $110 \%$ & $69 \%$ \\
\hline Overall (IQR)\% & $17 \%$ & $18 \%$ & $191 \%(49-380)$ & $183 \%(38-386)$ & $205 \%(58-396)$ & $205 \%(53-410)$ \\
\hline \multicolumn{7}{|l|}{ High income } \\
\hline United States & $0 \%$ & $0 \%$ & $468 \%$ & $414 \%$ & $466 \%$ & $414 \%$ \\
\hline Overall (IQR) & $0 \%$ & $0 \%$ & $468 \%(264-733)$ & $414 \%(175-695)$ & $466 \%(255-733)$ & $414 \%(171-698)$ \\
\hline
\end{tabular}

Median CD4\% at the Start of cART of Children Younger Than 5 Yrs

\begin{tabular}{|c|c|c|c|c|}
\hline \multirow[b]{3}{*}{ Country } & \multirow{2}{*}{\multicolumn{2}{|c|}{ Complete Case }} & & \\
\hline & & & \multicolumn{2}{|c|}{ Imputed Data } \\
\hline & Female & Male & Female & Male \\
\hline \multicolumn{5}{|l|}{ Low income } \\
\hline Benin & NA & NA & Excluded & Excluded \\
\hline Burkina Faso & $20 \%$ & $18 \%$ & Excluded & Excluded \\
\hline Cambodia & $12 \%$ & $11 \%$ & $12 \%$ & $11 \%$ \\
\hline Kenya & $13 \%$ & $12 \%$ & $13 \%$ & $13 \%$ \\
\hline Malawi & $18 \%$ & $14 \%$ & $17 \%$ & $14 \%$ \\
\hline Mali & $20 \%$ & $14 \%$ & $17 \%$ & $12 \%$ \\
\hline Mozambique & $14 \%$ & $13 \%$ & $14 \%$ & $13 \%$ \\
\hline Rwanda & NA & NA & $12 \%$ & $13 \%$ \\
\hline Tanzania & $12 \%$ & $20 \%$ & $13 \%$ & $21 \%$ \\
\hline Uganda & $12 \%$ & $14 \%$ & $12 \%$ & $14 \%$ \\
\hline Zimbabwe & $12 \%$ & $17 \%$ & $11 \%$ & $13 \%$ \\
\hline Overall (IQR) & $13 \%(10-18)$ & $13 \%(9-17)$ & $14 \%(10-18)$ & $13 \%(9-17)$ \\
\hline \multicolumn{5}{|c|}{ Lower middle income } \\
\hline Côte d'Ivoire & $14 \%$ & $14 \%$ & $14 \%$ & $15 \%$ \\
\hline Ghana & $14 \%$ & $14 \%$ & $12 \%$ & $14 \%$ \\
\hline India & $10 \%$ & $12 \%$ & Excluded & Excluded \\
\hline Indonesia & $13 \%$ & $7 \%$ & $13 \%$ & $6 \%$ \\
\hline
\end{tabular}


TABLE 2. (Continued) CD4 Cell Count and CD4\% at the Start of CART and the Percentage of Children Starting cART With Severe Immunodeficiency by Country and World Bank Income Group

\begin{tabular}{|c|c|c|c|c|}
\hline \multirow[b]{3}{*}{ Country } & \multicolumn{4}{|c|}{ Median CD4\% at the Start of cART of Children Younger Than 5 Yrs } \\
\hline & \multicolumn{2}{|c|}{ Complete Case } & \multicolumn{2}{|c|}{ Imputed Data } \\
\hline & Female & Male & Female & Male \\
\hline Senegal & $28 \%$ & $17 \%$ & $19 \%$ & $16 \%$ \\
\hline Vietnam & $11 \%$ & $9 \%$ & $11 \%$ & $9 \%$ \\
\hline Zambia & $17 \%$ & $15 \%$ & $17 \%$ & $15 \%$ \\
\hline Overall (IQR) & $16 \%(11-22)$ & $14 \%(9-20)$ & $16 \%(11-22)$ & $15 \%(10-20)$ \\
\hline \multicolumn{5}{|c|}{ Upper middle income } \\
\hline Botswana & $11 \%$ & $30 \%$ & Excluded & Excluded \\
\hline Brazil & $22 \%$ & $18 \%$ & $22 \%$ & $18 \%$ \\
\hline Malaysia & $17 \%$ & $17 \%$ & $17 \%$ & $15 \%$ \\
\hline Peru & $11 \%$ & $8 \%$ & Excluded & Excluded \\
\hline South Africa & $14 \%$ & $14 \%$ & $14 \%$ & $14 \%$ \\
\hline Thailand & $13 \%$ & $13 \%$ & $14 \%$ & $13 \%$ \\
\hline Overall (IQR) & $14 \%(9-21)$ & $14 \%(9-20.6)$ & $14 \%(9-21)$ & $14 \%(9-21)$ \\
\hline \multicolumn{5}{|l|}{ High income } \\
\hline United States & $31 \%$ & $28 \%$ & $31 \%$ & $28 \%$ \\
\hline Overall (IQR) & $31 \%(22-40)$ & $28 \%(19-34)$ & $31 \%(22-40)$ & $28 \%(19-34)$ \\
\hline
\end{tabular}

Percentage of Children Starting cART With Severe Immunodeficiency

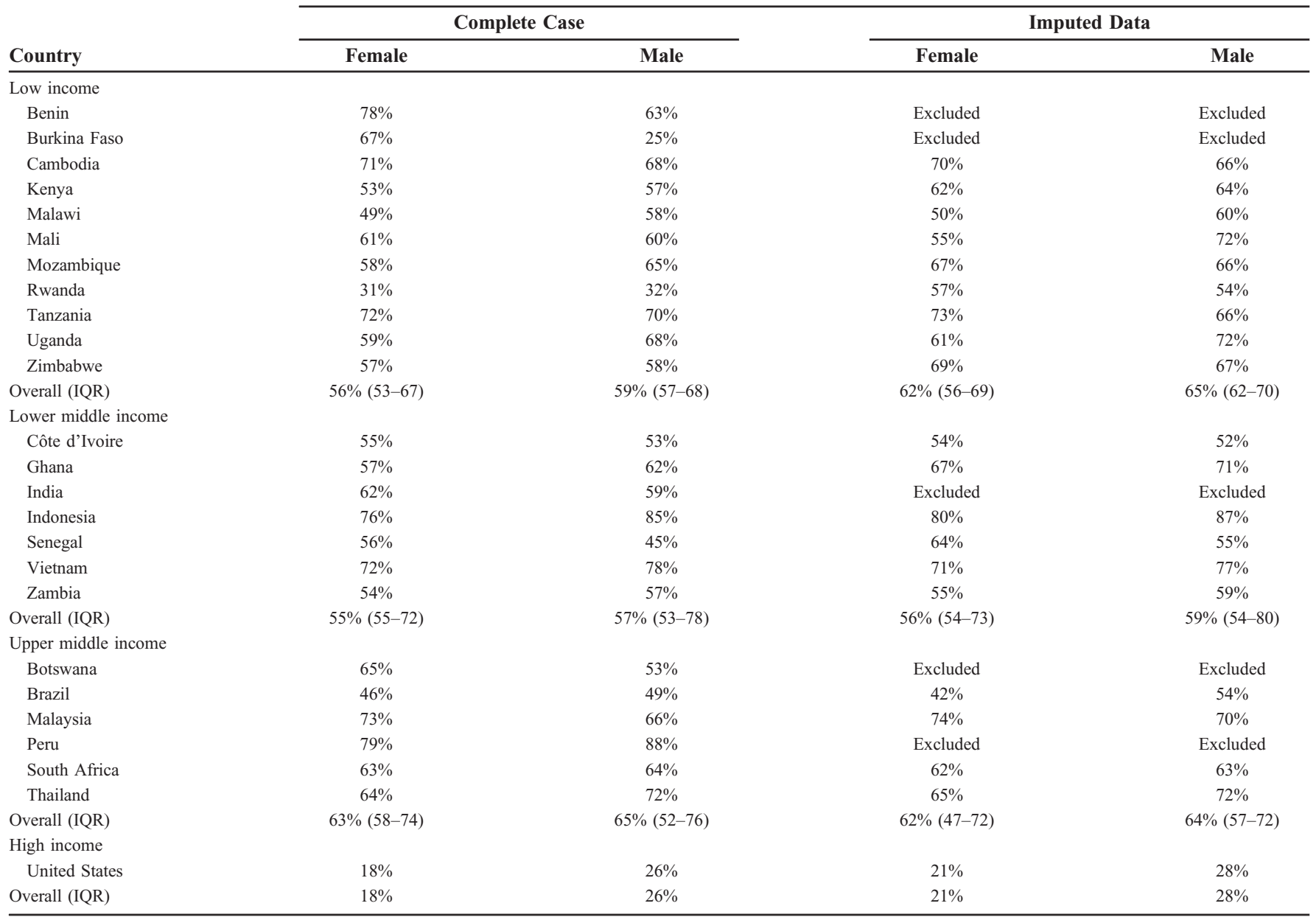

Analyses based on 11,125 children aged 5 years or older with CD4 counts and 6963 children below 5 years with CD4\% data (complete case) and 34,706 children after imputation of missing data. Complete case data includes all countries and calendar years. Imputed data only includes calendar years from individual countries with $\geq 10$ children with available CD4 measures and countries with $\geq 50$ children with available CD4 measures.

NA, not applicable (no data). 
microliter (girls from Uganda). For children aged $<5$ years, the differences ranged from $-1 \%$ (boys from Kenya) to $+4 \%$ (boys from Zimbabwe).

\section{Temporal Trends in CD4 Measures}

Data from low- and middle-income countries typically spanned the years 2003-2010, whereas data from the United States were available for 1995-2006 only (Table 1). We first fitted models including separate smoothers for each combination of gender, age group, and country income group. Gender did not improve model fit and was therefore removed. The final models included smoothers for each combination of age and country income-group. Figure 2 shows modeled temporal trends in the prevalence of severe immunodeficiency at cART initiation; Figure 3 shows corresponding trends in median CD4 counts (children aged $\geq 5$ years) or CD $4 \%$ (children $<5$ years). In LIC, the estimated percentage of children starting cART with severe immunodeficiency (as defined by WHO 2006 criteria) declined from $76 \%$ in 2004 to $63 \%$ in 2010. Corresponding figures for LMIC were from $77 \%$ to $66 \%$ and for UMIC from $75 \%$ to $58 \%$. In the United States, it decreased from $42 \%$ to $19 \%$ during 1996-2006 (Fig. 2).

In LIC, the median CD4 cell count at cART initiation in children aged $\geq 5$ years increased by $72 \%$ from 2004 to
2010, from 144 to 247 cells per microliter. Corresponding increases for LMIC were $20 \%(115-138$ cells $/ \mu \mathrm{L})$ and $52 \%$ in UMIC $(101-153$ cells $/ \mu \mathrm{L})$. The increase from 1996 to 2006 in the United States was 72\% (254-398 cells/ $\mu \mathrm{L})$. In children aged $<5$ years, CD4\% increased from 2004 to 2010 from $13 \%$ to $17 \%$ in LIC, from $11 \%$ to $15 \%$ in LMIC, and from $13 \%$ to $19 \%$ in UMIC. In the United States, CD4\% increased between 1996 and 2005 from 27\% to $32 \%$ (Fig. 3). Results of complete case analyses were similar (see Figure S3 and Figure S4, Supplemental Digital Content 5 and 6, http://links.lww.com/QAI/A579).

Temporal trends in median age at cART initiation varied between country income groups (see Figure S5, Supplemental Digital Content 7, http://links.lww.com/QAI/A579). There was no clear trend in median age at cART initiation in UMIC, whereas there was a decrease over time in LMIC (6.1-3.0 years) and LIC (6.5-4.6 years). In the United States, median age increased from 5.6 to 10.9 years (1996-2006).

\section{Determinants of Starting cART With Severe Immunodeficiency}

Table 3 shows the adjusted OR of starting cART with severe immunodeficiency by gender, age group, and
FIGURE 2. Severe immunodeficiency at the start of combination antiretroviral therapy by age and country income group. Results from generalized additive mixed-effects model based 34,706 children after imputation of missing data. $95 \% \mathrm{Cls}$ are shown as shaded areas.
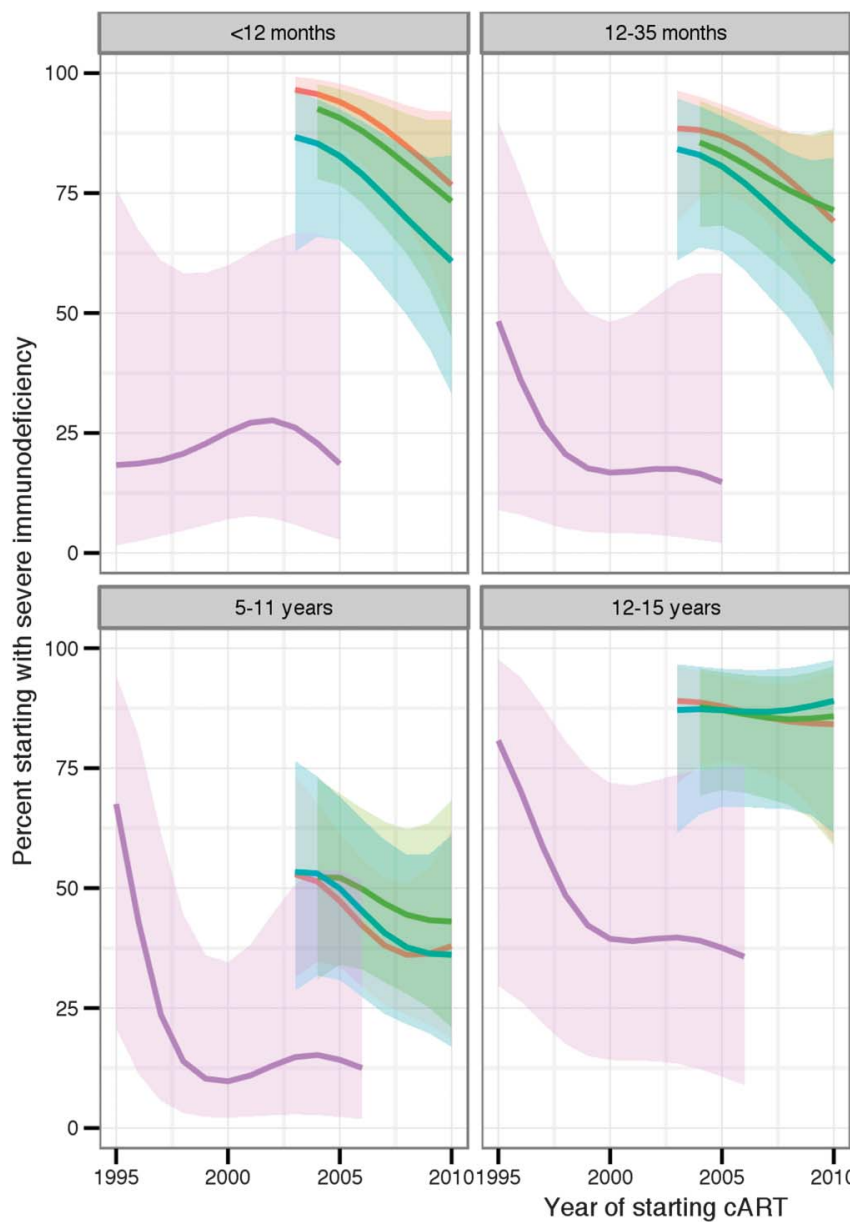


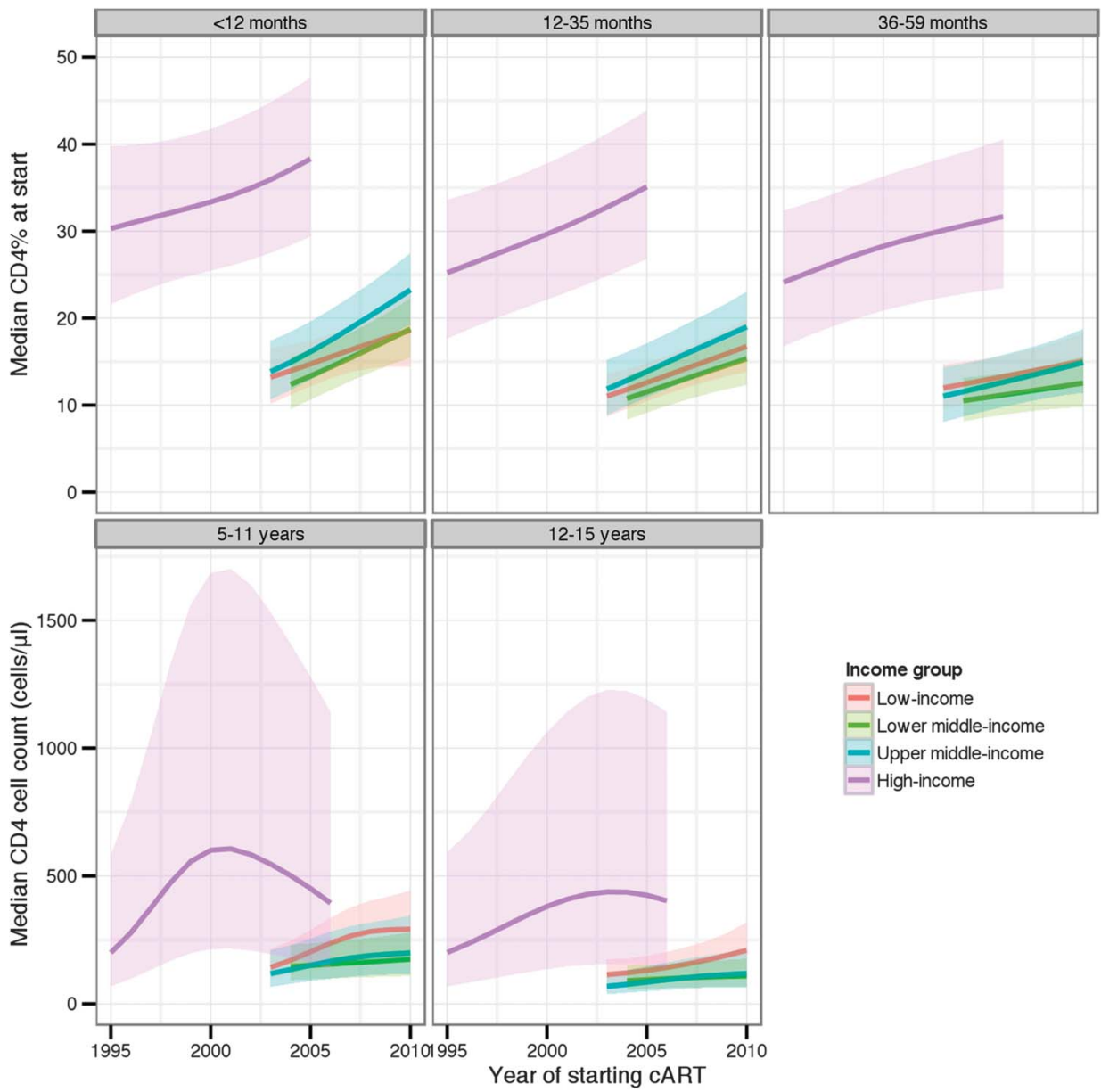

FIGURE 3. Median CD4 cell count in children aged 5 years or older and median CD4\% in children below 5 years of age at the start of combination antiretroviral therapy by age and country income group. Results from generalized additive mixed-effects model based on 34,706 children after imputation of missing data. 95\% Cls are shown as shaded areas.

income group in 2009 based on 4121 children from 4 LIC (Cambodia, Malawi, Rwanda, and Zimbabwe), 3 LMIC (Indonesia, Vietnam, and Zambia), and 4 UMIC (Brazil, Malaysia, South Africa, Thailand). There was no difference in the probability of starting cART with severe immunodeficiency between genders but the ORs comparing the risk in infants with older children decreased up to age 5-11 years (OR, $0.22 ; 95 \% \mathrm{CI}: 0.17$ to 0.28$)$ but increased in age group $12-15$ years (OR, 2.14; 95\% CI: 1.51 to 3.04). There was little evidence for a difference between country income groups, or across levels of national pediatric cART coverage. Results were similar in the complete case analysis (see Table S2, Supplemental Digital Content 8, http://links.lww.com/QAI/A579).

\section{DISCUSSION}

This global analysis of CD4 measures at cART initiation was based on over 35,000 children starting treatment in sub-Saharan Africa, South East Asia, Latin America, and North America. The percentage of children starting cART with severe immunodeficiency was substantially lower in the United States compared with other countries, with only small differences between low-income and middle-income countries. The estimated proportion of children starting cART with severe immunodeficiency decreased everywhere over the study period. In 2010, however, approximately two-thirds of children in low- and middle-income countries still started cART with severe immunodeficiency.

\section{Discussion of Findings in the Light of WHO HIV Treatment Guidelines}

Our study confirms previous observations that many children in low- and middle-income countries continue to start cART late. ${ }^{6,8,22-24}$ In $2010,70 \%$ of children $<2$ years started cART with severe immunodeficiency despite WHO guidelines recommending early cART regardless of immunologic or clinical thresholds for all children in this age group. ${ }^{7}$ This may be partly due to delayed country-level implementation of WHO guidelines as well as poor access to early infant diagnosis (EID), slow turn-around time of test results, and limited cART availability for infants and young children. ${ }^{25}$ There was, however, wide variability in median CD4 values for countries in the same income group. Outside the United States, the proportion of children starting cART with severe immunosuppression was similar across countries with different income levels. This may be due to substantial donor support for cART in LIC, in contrast to middle-income countries 
TABLE 3. Individual-Level and Country-Level Adjusted Predictors for Starting CART With Severe Immunodeficiency in 2009

\begin{tabular}{lcc}
\hline Variable & Adjusted OR & $\mathbf{9 5 \%}$ CIs \\
\hline Gender & & \\
$\quad$ Female & 1.00 & \\
$\quad$ Male & 1.09 & 0.92 to 1.29 \\
Age group (yrs) & & \\
$\quad<1$ & 1.00 & 0.59 to 1.05 \\
1 to $<3$ & 0.79 & 0.35 to 0.71 \\
3 to $<5$ & 0.50 & 0.17 to 0.28 \\
5 to $<12$ & 0.22 & 1.51 to 3.04 \\
12 to $<16$ & 2.14 & \\
Country income level & & \\
$\quad$ Low & 1.00 & 0.63 to 2.88 \\
Lower middle & 1.35 & 0.38 to 1.61 \\
$\quad$ Upper middle & 0.79 & \\
National cART coverage (\%)* & & \\
$\quad<40$ & 1.00 & 0.3 to 1.49 \\
40 to $<60$ & 0.67 & 0.64 to 2.91 \\
60 to $<80$ & 1.37 & 0.4 to 1.68 \\
$\geq 80$ & 0.82 & \\
\hline
\end{tabular}

Results from generalized linear mixed model based on 4121 children starting cART in 2009 from the countries Brazil, Cambodia, Indonesia, Malawi, Malaysia, Rwanda, South Africa, Thailand, Vietnam, Zambia, and Zimbabwe with missing values imputed using multiple imputation.

*Based on a separate analysis that included national pediatric cART coverage instead of country income level.

where national governments had to provide more finance for cART programs.

Although the median age at cART initiation decreased over time in LIC and LMIC, it increased in the United States, and there was no clear trend in UMIC, although the CIs were wide for all country income groups. Interpreting these trends is complex; the median age at cART initiation depends on the effectiveness of prevention of mother-tochild transmission (PMTCT) programs in reducing numbers of newly infected infants, capacity for EID and early cART, as well as the backlog of older children not yet on therapy, which may be substantial. ${ }^{26,27}$ For example, the increasing age over time in the United States is likely because effective PMTCT programs had largely eliminated new infant infections, with the few children initiating cART in later years being long-term survivors who had remained relatively healthy without cART.

It is hoped that 2013 guidelines recommending universal cART for all children aged $<5$ years irrespective of CD4 or clinical stage will accelerate pediatric cART access by simplifying programs to a single recommendation for all children in this age group. ${ }^{9}$ These recommendations not only expand treatment eligibility but could potentially accelerate treatment initiation even for those who actually do have immunosuppression by removing the requirement for a CD4 measurement and consequent time lag while waiting for results before treatment is started. Changes in CD4 measures at ART initiation may therefore be a useful measure of responsiveness to guideline changes, with targets set for reductions in the proportion of children starting cART with severe immunosuppression. ${ }^{28}$

\section{Role of Infant and Child Diagnostic Testing}

The scale-up of PMTCT programs including antenatal opt-out HIV testing and EID testing may have contributed to gradually higher CD4 cell measures at cART initiation in infants. If more women were aware of their HIV status in pregnancy and enrolled in PMTCT programs with access to EID, there would be increasing likelihood of earlier testing of their HIV-exposed infants. In addition, the scale-up of EID through dried blood spot HIV-PCR testing is encouraging. ${ }^{29-31}$ However, coverage remains low in many settings and postnatal PMTCT infant follow-up is frequently suboptimal, ${ }^{25,32}$ with many challenges such as lack of integration of PMTCT, HIV, and child health programs, limited privacy in routine child health clinics, and slow turn-around times of HIV-PCR test results. ${ }^{33,34} \mathrm{HIV}$-infected older children are also frequently only identified once they have advanced disease, highlighting the importance of integrating provider-initiated HIV serological testing (cheap and easy to perform) at routine child health visits, especially in high prevalence settings. ${ }^{35,36}$

\section{Poor Access to CART for Infants and Children}

Timely diagnosis of pediatric HIV does not necessarily result in timely cART. In national programs in Cambodia, Namibia, Senegal, and Uganda, despite impressive EID scaleup, the proportion of infants identified as infected who subsequently initiated cART was $<40 \%$ in all countries except Namibia. ${ }^{29}$ Key reasons for the diagnosis to treatment gap include HIV diagnostic tests and pediatric cART being located at separate sites without robust referral mechanisms between services, challenges with CD4 measurement to determine eligibility including access to tests, turn-around time and interpretation of results, as well as health care worker discomfort with treating children. ${ }^{29}$ In a review of studies including mostly older children, the proportion of cART eligible children that initiated treatment was as low as $40 \%{ }^{37}$ Approaches such as decentralization of pediatric cART to maternal and child health and clinics together with taskshifting and health care worker training in pediatric treatment and care may facilitate earlier cART initiation. ${ }^{38}$

\section{Strengths and Limitations}

This is a large pooled individualized patient-level data set from multiple programs across the globe. In contrast to aggregate reporting data, it provides a more nuanced picture of progress toward pediatric cART access in a range of settings. However, we could not examine trends after 2010 because more recent data were not yet available from many sites. This is important as most lower income countries implemented the WHO 2010 recommendation of ART initiation irrespective of disease severity in children aged $<2$ years in 2011, and until recently, there was limited access to EID. ${ }^{21,31}$ There was substantial missing data on CD4 measures and clinical stage from low- and middle-income countries, so our estimates of median CD4 values may be biased. The high proportion of missing CD4 and clinical stage data is a concern as, for most of the data collection period, these measures were required to assess cART eligibility; their poor 
availability may indicate missed opportunities to initiate cART. In this respect, the recent WHO recommendation of cART initiation in all children aged $<5$ years may improve pediatric cART access by eliminating the requirement for these measures before cART initiation but may also preclude repeating analyses such as this one, making it more difficult to accurately monitor progress in access to cART. ${ }^{8}$ Data from some countries were limited to a small number of patients from a single clinic and so were excluded from analyses of time trends and predictors of CD4 at cART initiation as they are unlikely to be nationally representative. There were data from only 1 high-income country (United States), with relatively small numbers of children. In addition, only data up to 2005 from the United States could be analyzed precluding comparison with low- and middle-income countries during the major period of scale-up in the latter regions.

\section{CONCLUSIONS}

Reductions in the proportion of children initiating cART with severe immunosuppression in low- and middleincome countries over time are encouraging but modest. Efforts to improve timely access to pediatric HIV diagnosis and cART should remain a global public health priority.

\section{ACKNOWLEDGMENTS}

The authors are grateful to all children, care givers, and data managers involved in the participating cohorts and treatment programs.

\section{REFERENCES}

1. World Health Organization UNAIDS UNICEF. Global HIV/AIDS Response: Epidemic Update and Health Sector Progress towards Universal Access: Progress Report 2011. 2011. Available at: http://www. who.int/hiv/pub/progress_report2011/en/index.html. Accessed June 7, 2012.

2. World Health Organization. Global Update on the Health Sector Response to HIV, 2014. Geneva, Switzerland; 2014. Available at: http://www.who.int/hiv/pub/progressreports/en/. Accessed July 21, 2014.

3. Bolton-Moore C, Mubiana-Mbewe M, Cantrell RA, et al. Clinical outcomes and CD4 cell response in children receiving antiretroviral therapy at primary health care facilities in Zambia. JAMA. 2007;298:1888-1899.

4. Sutcliffe CG, van Dijk JH, Bolton C, et al. Effectiveness of antiretroviral therapy among HIV-infected children in sub-Saharan Africa. Lancet Infect Dis. 2008;8:477-489.

5. Davies MA, Keiser O, Technau K, et al. Outcomes of the south african national antiretroviral treatment programme for children: the IeDEA southern africa collaboration. S Afr Med J. 2009;99:730-737.

6. Leroy V, Malateste K, Rabie H, et al. Outcomes of antiretroviral therapy in children in Asia and Africa: a comparative analysis of the IeDEA pediatric multiregional collaboration. J Acquir Immune Defic Syndr. 2013;62:208-219.

7. World Health Organization. Antiretroviral therapy of HIV infection in infants and children: towards universal access: recommendations for a public health approach-2010 revision. 2010;1-194. Available at: http://www.who.int/hiv/pub/paediatric/infants2010/en/index.html. Accessed July 26, 2011.

8. Davies M, May M, Bolton-Moore C, et al. Prognosis of children with HIV-1 infection starting antiretroviral therapy in Southern Africa: a collaborative analysis of treatment programs. Pediatr Infect Dis J. 2014;33: 608-616.

9. World Health Organization. Consolidated Guidelines on the Use of Antiretroviral Drugs for Treating and Preventing HIV Infection.
Summary of Key Features and Recommendations. 2013. Available at: http://www.who.int/hiv/pub/guidelines/arv2013/short_summary/ en/index.html. Accessed February 11, 2014.

10. Wettstein C, Mugglin C, Egger M, et al. Missed opportunities to prevent mother-to-child-transmission in sub-Saharan africa: systematic review and meta-analysis. AIDS. 2012;26:2361-2373.

11. Gange SJ, Kitahata MM, Saag MS, et al. Cohort profile: the North american AIDS cohort collaboration on research and design (NAACCORD). Int J Epidemiol. 2007;36:294-301.

12. McGowan CC, Cahn P, Gotuzzo E, et al. Network for HIV research (CCASAnet) collaboration within the International Epidemiologic Databases to Evaluate AIDS (IeDEA) programme. Int J Epidemiol. 2007;36:969-976.

13. Zhou J, Kumarasamy N, Ditangco R, et al. The TREAT Asia HIV Observational Database: baseline and retrospective data. $J$ Acquir Immune Defic Syndr. 2005;38:174-179.

14. The IeDEA Pediatric Working Group. A survey of paediatric HIV programmatic and clinical management practices in Asia and sub-Saharan Africa-the International epidemiologic Databases to Evaluate AIDS (IeDEA). J Int AIDS Soc. 2013;16:17998.

15. Hazra R, Stoszek SK, Hance LF, et al. Cohort Profile: NICHD International Site Development Initiative (NISDI): a prospective, observational study of HIV-exposed and HIV-infected children at clinical sites in Latin American and Caribbean countries. Int J Epidemiol. 2009;38:1207-1214.

16. Van Dyke RB, Patel K, Siberry GK, et al. Antiretroviral treatment of US children with perinatally acquired HIV infection: temporal changes in therapy between 1991 and 2009 and predictors of immunologic and virologic outcomes. J Acquir Immune Defic Syndr. 2011 57:165-173.

17. Patel K, Ming X, Williams PL, et al. International Maternal Pediatric Adolescent ACTCST. Impact of HAART and CNS-penetrating antiretroviral regimens on HIV encephalopathy among perinatally infected children and adolescents. AIDS. 2009;23:1893-1901.

18. The World Bank. How we classify countries. Available at: http://data worldbank.org/about/country-classifications. Accessed December 5, 2012.

19. World Health Organization. Antiretroviral therapy for HIV Infection in adults and adolescents in resource-limited settings: towards universal access. Recommendations for a Public Health Approach. 2006. Available at: http://www.who.int/hiv/pub/guidelines/artadultguidelines.pdf Accessed July 27, 2007

20. World Health Organization. Towards universal access. Scaling up priority HIV/AIDS interventions in the health sector. Prog Rep. 2010. 2010. Available at: http://www.who.int/hiv/pub/2010progressreport/en/index.html. Accessed May 8, 2012.

21. Rubin DB. Multiple Imputation for Nonresponse in Surveys. New York, NY: John Wiley; 1987.

22. Fatti G, Bock P, Eley B, et al. Temporal trends in baseline characteristics and treatment outcomes of children starting antiretroviral treatment: an analysis in four provinces in South Africa, 2004-2009. J Acquir Immune Defic Syndr. 2011;58:e60-67.

23. Peacock-Villada E, Richardson BA, John-Stewart GC. Post-HAART outcomes in pediatric populations: comparison of resource-limited and developed countries. Pediatrics. 2011;127:e423-441.

24. McNairy ML, Lamb MR, Carter RJ, et al. Retention of HIV-infected children on antiretroviral treatment in HIV care and treatment programs in Kenya, Mozambique, Rwanda, and Tanzania. J Acquir Immune Defic Syndr. 2013;62:e70-81

25. UNAIDS. 2013 Progress report on the global plan towards the elimination of new HIV infection in children by 2015 and keeping their mothers alive. 2013. Available at: http://www.unaids.org/en/media/unaids/contentassets documents/unaidspublication/2013/20130625_progress_global_plan_en.pdf. Accessed June 25, 2013

26. Ferrand R, Lowe S, Whande B, et al. Survey of children accessing HIV services in a high prevalence setting: time for adolescents to count? Bull World Health Organ. 2010;88:428-434.

27. Ferrand RA, Munaiwa L, Matsekete J, et al. Undiagnosed HIV infection among adolescents seeking primary health care in Zimbabwe. Clin Infect Dis. 2010;51:844-851.

28. Johnson LF, Boulle A. How should access to antiretroviral treatment be measured? Bull World Health Organ. 2011;89:157-160.

29. Chatterjee A, Tripathi S, Gass R, et al. Implementing services for Early Infant Diagnosis (EID) of HIV: a comparative descriptive 
analysis of national programs in four countries. BMC Public Health. 2011;11:553.

30. Sherman GG, Cooper PA, Coovadia AH, et al. Polymerase chain reaction for diagnosis of human immunodeficiency virus infection in infancy in low resource settings. Pediatr Infect Dis J. 2005;24:993-997.

31. Yapo V, d'Aquin Toni T, Desmonde S, et al. Evaluation of dried blood spot diagnosis using HIV1-DNA and HIV1-RNA Biocentric assays in infants in Abidjan, Cote d'Ivoire. The Pedi-test DBS ANRS 12183 study. J Virol Methods. 2013;193:439-445.

32. Sibanda E, Weller I, Hakim J, et al. The magnitude of loss to follow-up of HIV-exposed infants along the prevention of mother-to-child HIV transmission continuum of care: a systematic review and meta-analysis. AIDS. 2013;27:2787-2797.

33. Dube Q, Dow A, Chirambo C, et al. Implementing early infant diagnosis of HIV infection at the primary care level: experiences and challenges in Malawi. Bull World Health Organ. 2012;90:699-704.
34. Seidenberg P, Nicholson S, Schaefer M, et al. Early infant diagnosis of HIV infection in Zambia through mobile phone texting of blood test results. Bull World Health Organ. 2012;90:348-356.

35. Rollins N, Mzolo S, Moodley T, et al. Universal HIV testing of infants at immunization clinics: an acceptable and feasible approach for early infant diagnosis in high HIV prevalence settings. AIDS. 2009;23:1851-1857.

36. Weigel R, Kamthunzi P, Mwansambo $C$, et al. Effect of providerinitiated testing and counseling and integration of ART services on access to HIV diagnosis and treatment for children in Lilongwe, Malawi: a pre- post comparison. BMC Pediatr. 2009;9:80.

37. Mugglin C, Wandeler G, Estill J, et al. Retention in care of HIV-infected children from HIV test to start of antiretroviral therapy: systematic review. PLoS One. 2013;8:e56446.

38. Penazzato M, Davies M, Apollo T, et al. Task shifting for the delivery of pediatric antiretroviral treatment: a systematic review. J Acquir Immune Defic Syndr. 2014;65:414-422. 